\title{
Comparison of class 2 transposable elements at superfamily resolution reveals conserved and distinct features in cereal grass genomes
}

\author{
Yujun Han ${ }^{1}$, Shanshan Qin ${ }^{1}$ and Susan R Wessler ${ }^{1,2^{*}}$
}

\begin{abstract}
Background: Class 2 transposable elements (TEs) are the predominant elements in and around plant genes where they generate significant allelic diversity. Using the complete sequences of four grasses, we have performed a novel comparative analysis of class 2 TEs. To ensure consistent comparative analyses, we re-annotated class 2 TEs in Brachypodium distachyon, Oryza sativa (rice), Sorghum bicolor and Zea mays and assigned them to one of the five cut-and-paste superfamilies found in plant genomes (TC1/mariner, PIF/Harbinger, hAT, Mutator, CACTA). We have focused on noncoding elements because of their abundance, and compared superfamily copy number, size and genomic distribution as well as correlation with the level of nearby gene expression.

Results: Our comparison revealed both unique and conserved features. First, the average length or size distribution of elements in each superfamily is largely conserved, with the shortest always being TC1/mariner elements, followed by PIF/Harbinger, hAT, Mutator and CACTA. This order also holds for the ratio of the copy numbers of noncoding to coding elements. Second, with the exception of CACTAs, noncoding TEs are enriched within and flanking genes, where they display conserved distribution patterns, having the highest peak in the promoter region. Finally, our analysis of microarray data revealed that genes associated with TC1/mariner and PIF/Harbinger noncoding elements have significantly higher expression levels than genes without class 2 TEs. In contrast, genes with CACTA elements have significantly lower expression than genes without class 2 TEs.

Conclusions: We have achieved the most comprehensive annotation of class 2 TEs to date in these four grass genomes. Comparative analysis of this robust dataset led to the identification of several previously unknown features of each superfamily related to copy number, element size, genomic distribution and correlation with the expression levels of nearby genes. These results highlight the importance of distinguishing TE superfamilies when assessing their impact on gene and genome evolution.
\end{abstract}

Keywords: Genome comparison, Plant genomes, Genome evolution, Class2 transposable elements, Features, Grass genomes

\section{Background}

Transposable elements (TEs) are DNA fragments that can move from one genomic location to another by a process called transposition. They are the largest component of most multicellular organism genomes, often exceeding $50 \%$ of content $[1,2]$. TEs are divided into two classes based on the nature of their transposition intermediate: class 1 (RNA) retrotransposons and class 2 DNA transposons. If

\footnotetext{
* Correspondence: susan.wessler@ucr.edu

${ }^{1}$ Department of Plant Biology, University of Georgia, Athens, GA 30602, USA

${ }^{2}$ Department of Botany and Plant Sciences, University of California, Riverside, CA 92521, USA
}

\section{Biomed Central}

(c) 2013 Han et al.; licensee BioMed Central Ltd. This is an Open Access article distributed under the terms of the Creative Commons Attribution License (http://creativecommons.org/licenses/by/2.0), which permits unrestricted use, distribution, and reproduction in any medium, provided the original work is properly cited. we think of the genomes of higher plants as ecosystems, then each TE class occupies a characteristic niche. Although class 1 TEs are largely intergenic, most class 2 TEs are preferentially found in and around genes. As such, class 1 elements contribute more significantly to plant genome size differences whereas class 2 elements are frequently involved in generating allelic diversity [3].

Transposition of class 2 elements, which are the focus of this study, requires the enzyme transposase that binds to the elements' terminal inverted repeat (TIR) and catalyzes both excision and integration into a new site. Class 2 elements are classified into superfamilies based on the 
relatedness of the transposase and on shared structural features including the TIR sequence and the length of the target site duplication (TSD) that flanks the TIR and is generated during integration. Only five of the seventeen superfamilies characterized to date have been found in plant genomes (CACTA, Mutator, PIF/Harbinger, $h A T, T c 1 /$ mariner) $[4,5]$. While helitrons were considered to be the sixth class 2 superfamily in plants [6], we do not include them here because they have different structures and transposition mechanisms from cut-and-paste elements. Each superfamily contains autonomous and nonautonomous elements. Autonomous elements encode the transposase necessary for their own movement and the movement of nonautonomous superfamily members, which lack functional transposase genes.

The most numerous class 2 elements in characterized plant genomes and in several animal species are miniature inverted-repeat transposable elements (MITEs). MITEs are nonautonomous TEs that are characterized by short length (most $<600 \mathrm{bp}$ ), high sequence similarity, and a potential for very high copy number (hundreds or thousands) [7-9]. Despite lacking coding sequences, MITEs can be classified into known superfamilies based on related TIR sequence and TSD length. To date, the majority of reported MITEs are either Stowaway or Tourist elements that belong to the Tc1/mariner and PIF/Harbinger superfamilies, respectively [10-13]. MITEs belonging to the $h A T$ and the Mutator superfamilies have also been reported [14-17].

Although TE superfamilies can be readily distinguished by shared sequences and structural features (such as TIRs and TSDs), TEs are usually lumped together when their relationship with gene and genome evolution is analyzed $[18,19]$. A determination of potentially unique and conserved features of each superfamily would require their systematic comparison across species. The availability of complete genome sequences from four grass species, Brachypodium distachyon (250Mb) [20], Oryza sativa (rice)(340Mb) [21], Sorghum bicolor (750Mb) [22], and Zea mays (2500Mb) [1] has facilitated such a novel comparative analysis. These four grass species were chosen for several reasons. First, the genomes have been annotated to high quality and some gene expression data is available. Although TEs have also been annotated in these genomes, we performed a systematic re-annotation to permit a consistent comparative analysis and to classify noncoding elements into superfamilies. With only five of the $\sim 20$ class 2 cut-and-paste superfamilies identified in eukaryotes, it is more likely that most TEs in the genomes can be assigned. In contrast, with over 17 superfamilies, it is very difficult to classify Aedes aegypti TEs into superfamilies unambiguously [2]. In this study, we identified and classified over 450,000 class 2 elements. Finally, the genes in these four species are largely syntenic despite a 10-fold difference in genome size. Given that most class 2 elements are known to insert into or near genes, we were particularly interested in comparing each TE superfamily across species to determine what features are conserved and what features may be influenced by the host.

\section{Results}

\section{TE annotation and definitions}

Class 2 TEs from the five superfamilies found in plants were annotated in four grass genomes and the results are summarized in Figure 1 and Additional file 1. Coding elements contain all or part of the transposase gene from one of the five superfamilies. Their copy numbers were estimated from the number of conserved transposase domains identified by TARGeT (Figure $1 \mathrm{~A}$ and Additional file 1) [22]. We call them coding elements rather than the more conventional autonomous elements because the latter term implies functional activity, and manual curation of a subset of these elements indicates that most contain inactivating mutations. To save words, coding TEs are denoted with a lowercase " $c$ " followed by the superfamily name (e.g. the generics cTE as in $c C A C T A$ ). Similarly, noncoding elements are referred to as nTEs. In this study nTEs were discovered by MITE-Hunter, a structure based TE identification tool that has as its output consensus sequences that represent nTE families. By default MITE-Hunter identifies nTEs shorter than $2 \mathrm{~kb}$, which encompasses the majority of nTEs including MITEs [23].

To obtain the copy number, length and position of nTEs in each genome, consensus sequences were used as queries for searches using RepeatMasker. From the RepeatMasker output, double-ended elements and single-ended elements were identified and analyzed separately (see Methods for details). The total copy number of a nTE consensus sequence was calculated by adding the doubleended copy number and half of the single-ended copy number. Furthermore, nTEs were characterized as either MITE or non-MITE where MITEs are defined as shorter than $600 \mathrm{bp}$ with at least 25 double-ended copies or 10 nearly identical copies (identity $>=99 \%$ ) (Additional file 1 ). The total copy number and length of nTEs (Figure 1B and $C$ ) as well as the percentage of each superfamily in the four genomes (Figure 1D) was determined. While these data are combined in Figure 1 and Additional file 1, the complete dataset is available at http://target.iplant collaborative.org.

Copy numbers of class 2 elements in four grass genomes Except for the Tc1/mariner superfamily, the copy numbers of cTEs differ dramatically among the four grass genomes (Figure 1A and Additional file 1). cCACTAs are the most numerous in rice $(340 \mathrm{Mb})$, sorghum $(750 \mathrm{Mb})$ and maize $(2500 \mathrm{Mb})$ and are the second most numerous 


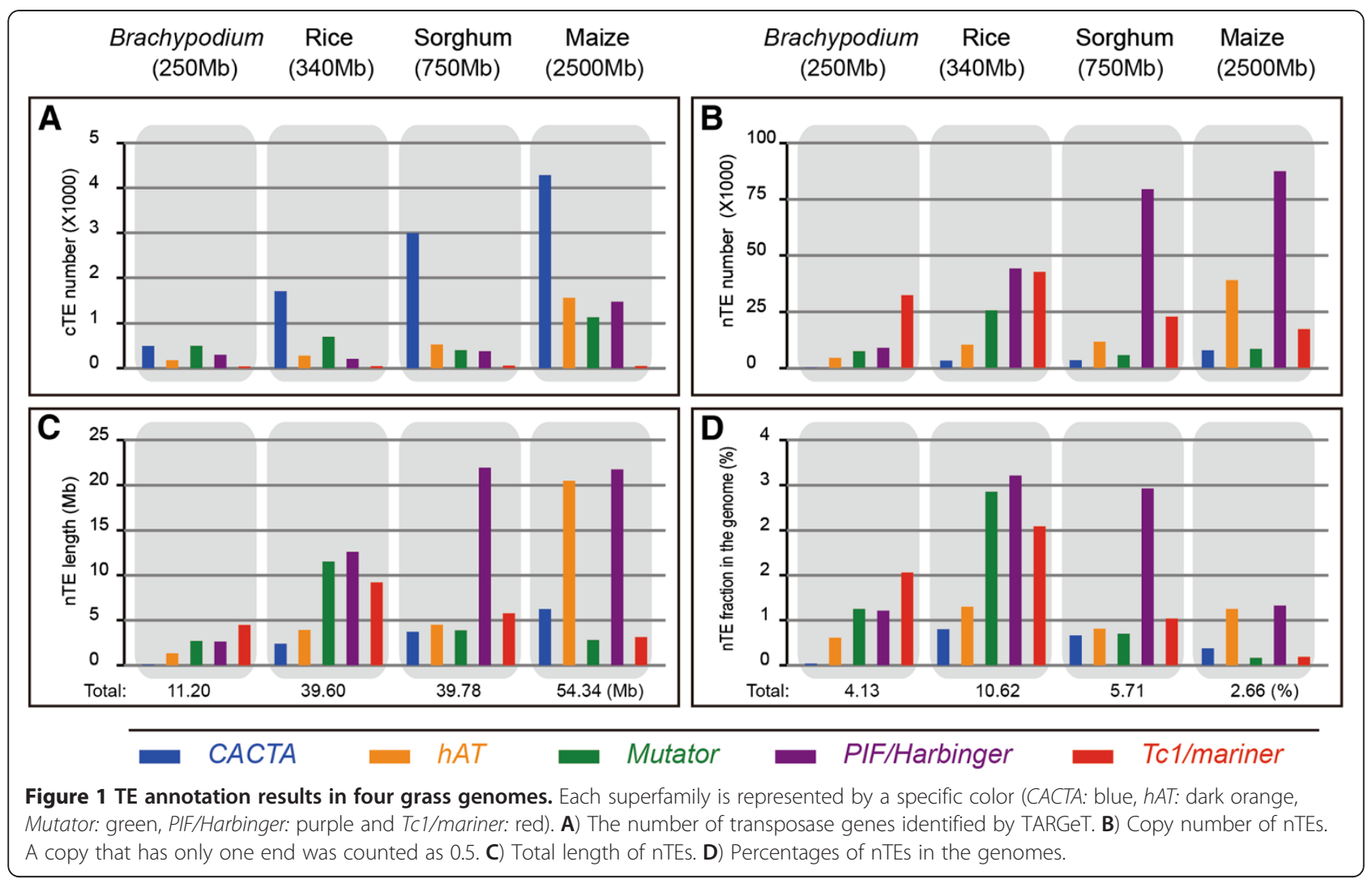

(after Mutator) in the Brachypodium genome (250Mb). Furthermore, $c$ CACTAs increase in copy number with increasing genome size (491 to 1705 to 2996 to 4288). chATs also increase with genome size but their numbers are significantly lower than CCACTAs (176, 277, 526, 1556). Coding members of the Mutator and PIF/Harbinger superfamilies show little copy number variation in Brachypodium, rice and sorghum but show a marked increase in the larger maize genome. Interestingly, the coding members of the Tc1/mariner superfamily have by far the lowest copy numbers in the four genomes and show no correlation with genome size (36, $45,57,47)$.

Despite the dramatic copy number differences across species, the copy number ratio of nTEs to CTEs is a conserved feature of each superfamily (Additional file 1). For each genome, the ratio is lowest for the CACTA superfamily (1.27 average ratio), which has the most cTEs and the fewest nTEs. After CACTA, the next lowest ratio in all genomes is for Mutator (18.14) followed by $h A T$ (27.16), PIF/Harbinger (128.53) and Tc1/mariner (647.25). The high ratios for PIF/Harbinger and Tc1/ mariner reflect the success of MITEs from these superfamilies, called Tourist and Stowaway, respectively [7,8]. These results indicate that the mechanisms underlying the generation and success of nTEs are both conserved and distinctive for each superfamily.

\section{Length is a conserved feature of the nTEs in a superfamily}

Figure 2 is a visual representation of the size and copy number of the nTEs from Tc1/mariner and PIF/Harbinger in the four genomes (the results of all superfamilies except CACTA are in Additional file 2). These data were generated by plotting the double-ended copy numbers of each consensus nTE against the length of the consensus sequence. Of note is the similarity of the patterns for a particular nTE superfamily in all genomes. In addition, with only one exception (nhAT and nMutator in maize), the order of the mean lengths of the nTEs is the same in all genomes with $n T c 1 /$ mariner $<n P I F /$ Harbinger $<n h A T<n M$ utator.

\section{All $n$ TEs except $n$ CACTAs are enriched near genes}

Prior studies showed that most plant class 2 TEs have a preference for insertion into or near genes [3,24,25]. Recently, the determination of over 800 insertions of the active MITE $m$ Ping near rice genes identified a preference for insertion of this member of the PIF/Harbinger superfamily within $1 \mathrm{~kb}$ of the transcription start site (TSS) and $1 \mathrm{~kb}$ downstream of the transcription stop site (TTS) [26]. Here our annotation results have been used to determine whether nTEs from other superfamilies show similar enrichment and, if so, whether this feature is conserved in all genomes. 

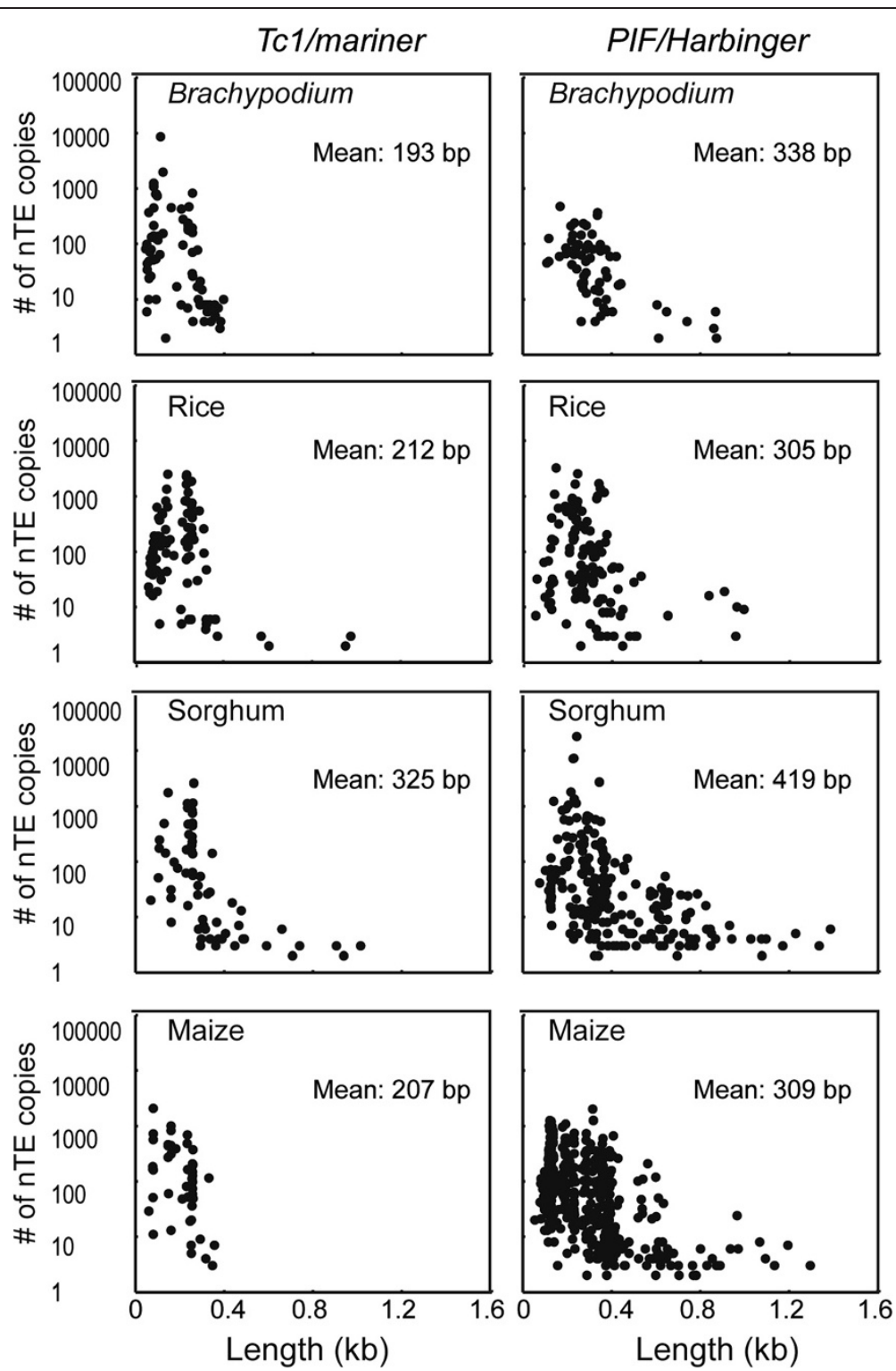

Figure 2 The double-ended copy number and length of the consensus nTEs of Tc1/mariner and PIF/Harbinger in Brachypodium, rice, sorghum and maize. The $y$-axis is in $\log 10$ scale.

To this end we first calculated the average percentages of class 2 nTEs in the whole genome as well as in the $5^{\prime}$ and $3^{\prime}$ flanking regions around genes. These data are presented diagrammatically in Figure 3A and in detail in Additional file 3 (this file also contains the distributions of all nTEs in the exons and introns, which is not discussed further). Taken together these data indicate that the nTEs from all superfamilies except CACTA are enriched in the $1 \mathrm{~kb}$ compartments flanking the $5^{\prime}$ and $3^{\prime}$ ends of coding regions. The only exception is in maize where there are more $n C A C T A s$ near genes than in other areas of the maize genome, which may reflect the overwhelming number of class 1 LTR elements that are enriched in intergenic regions (composing $74.6 \%$ of the maize genome [1]) [3]. The extent of enrichment is especially pronounced in rice, sorghum and maize where about $20 \%$ of the sequences in these regions are derived from class 2 nTE sequences (Figure $3 \mathrm{~A}$ and Additional file 3).

To characterize the distribution of nTEs at higher resolution and in regions more distant from the TSS and TTS, we calculated nTE percentages in continuous $10 \mathrm{bp}$ windows extending $10 \mathrm{~kb}$ upstream and $10 \mathrm{~kb}$ downstream of coding regions. The distributions of all nTEs in these regions are similar as evidenced by the black curves in Figure 3B, although the percentage of 


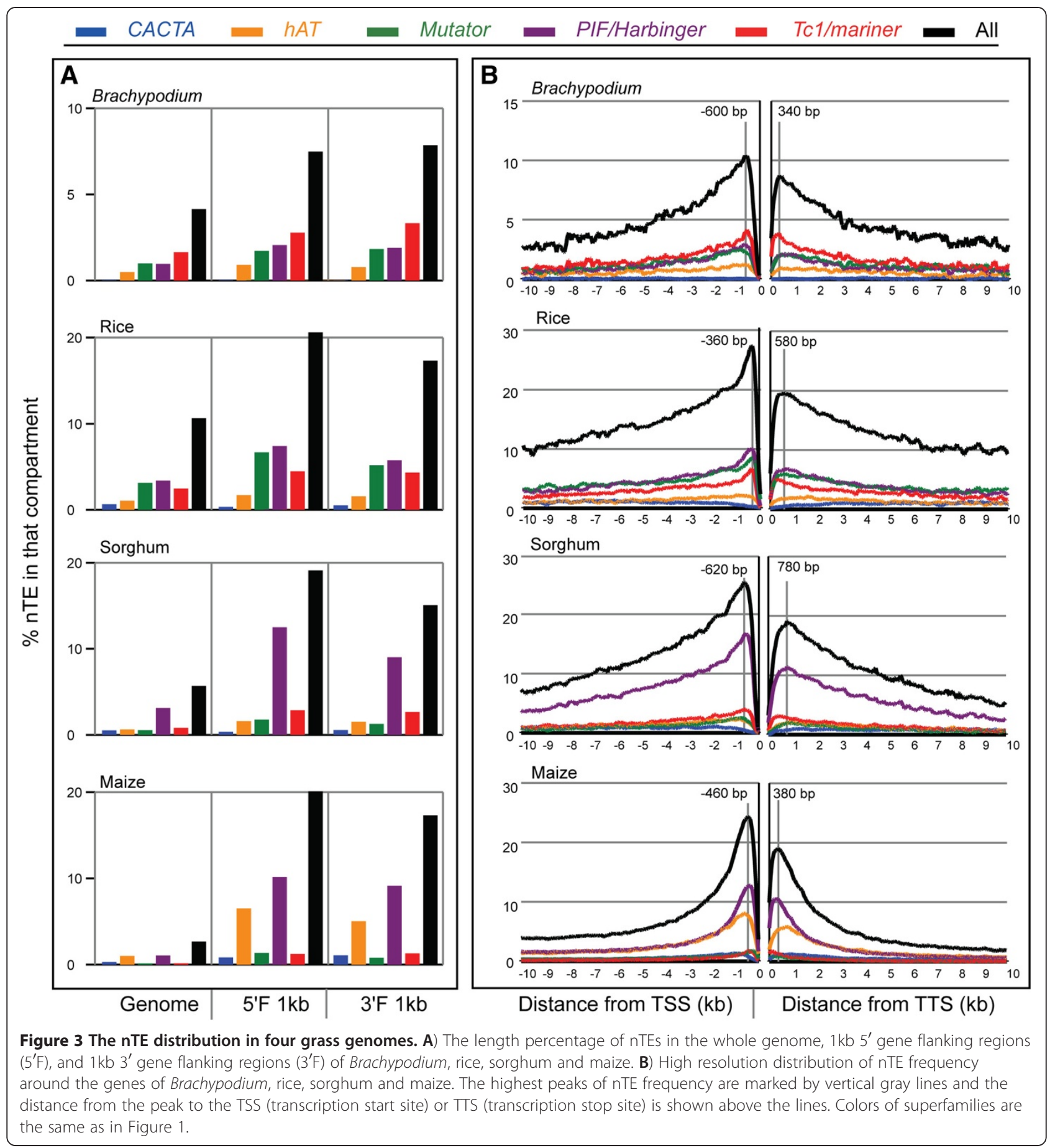

elements around the genes of Brachypodium is about half that of the other three species. After the $5^{\prime}$ and $3^{\prime}$ flanking regions, intron nTEs are the most abundant across all the superfamilies and species. However, unlike the patterns observed in the gene flanking regions, nTE frequencies are highest in the middle of introns and drop gradually toward the splice sites (see Additional file 4).
nTEs differ in their association with genes of high or low expression

The abundance of class 2 nTEs in genic regions prompted us to examine whether the presence of a particular superfamily member near genes correlates with increased or reduced expression levels. This analysis was performed in rice and maize where annotation is of high quality and gene expression data is available. Genes 
harboring nTEs from members of the same TE superfamily were grouped and their expression levels were compared with a control group of genes without class 2 nTEs. Extensive comparisons were performed using rice microarray data from different experiments, tissues and platforms. A more limited comparison was also made with available maize expression data (see Methods for details).

In all microarray data analyzed in rice and maize, genes with $n P I F /$ Harbinger and $n T c 1 /$ mariner elements have significantly higher expression levels than genes in the control group. In contrast, the expression of both rice and maize genes with $n C A C T A s$ is significantly lower than the control dataset (Figure 4 and Additional file 5). Although expression levels for maize genes associated with nhATs and nMutators are significantly higher than the controls in RNA samples from two tissues (Figure $4 \mathrm{C}$ and D), no clear picture emerged when rice samples were analyzed (Figure 4A and B, Additional file 5 GSE23918).

To confirm these findings, we analyzed publically available RNA-seq data from rice and obtained very similar results (see Additional file 6). Specifically, genes with class 2 superfamily nTEs except for $n C A C T A s$, showed significantly higher expression levels than controls. The expression levels of genes with $n C A C T A s$ are lower than controls, but these data are not statistically significant ( $\mathrm{p}$-value $=0.0608$ ), which can be explained by

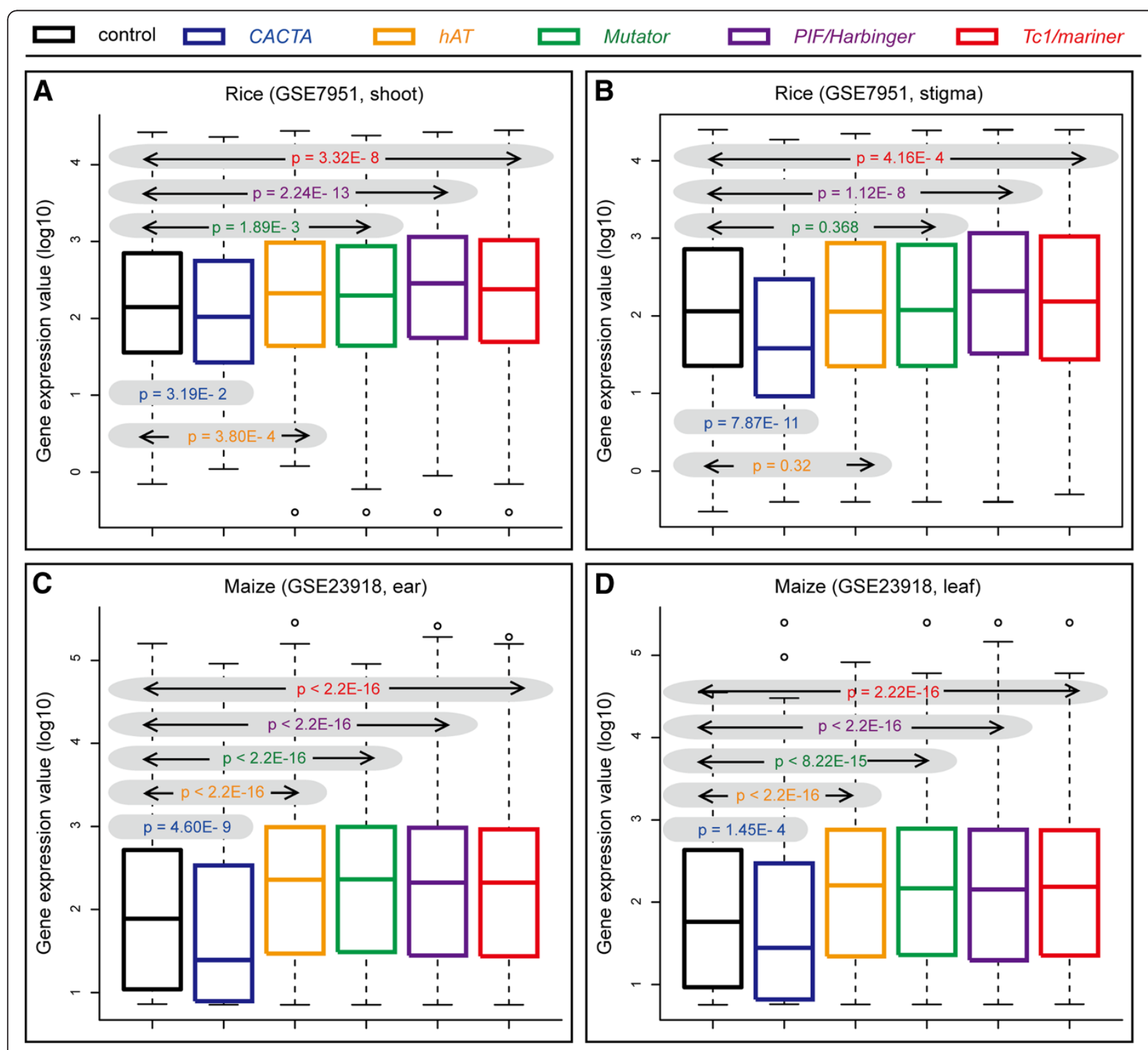

Figure 4 Comparison between the expression levels of genes with class 2 TEs and without class 2 TEs, in rice root (A) and stigma (B), maize ear (C) and leaf (D). The $y$-axis is log10 gene expression values. P-values of pairwise comparison are shown in shadow areas. Colors of superfamilies are the same as in Figure 1. 
the small sample size and limited sequencing reads to discern genes with low expression.

\section{Discussion}

The major focus of this study was to generate comprehensive and accurate class $2 \mathrm{TE}$ data for comparative analyses. To this end we utilized TARGeT and MITEHunter, two programs that have proved efficient at detecting cTEs and nTEs, respectively [22,23]. In our analyses we separated nTEs from cTEs, classified them into superfamilies, and further identified MITEs among the nTEs. This protocol was necessary because nTEs and cTEs have distinct features. For example, coding Tc1/mariners have only about 50 copies in each of the analyzed genome but there are two orders of magnitude more nTc1/mariners (mainly Stowaways) (Figure 1 and Additional file 1). The dramatic amplification potential of small nTEs, in particular MITEs, is also very different across superfamilies. Extensive manual curation was performed for each nTE consensus sequence to ensure the accuracy of TE discovery and classification. In this way, we achieved the most comprehensive annotation of class 2 cut-and-paste TEs to date in these four grass genomes. For example, we found several-fold more Stowaway and Tourist elements than a previous annotation of these elements in rice, sorghum and maize [22]. Comparative analysis of this robust dataset led to the identification of several previously unknown features related to copy number, element size, genomic distribution and correlation with the expression level of nearby genes.

The CACTA superfamily is the outlier in all comparisons. Among the superfamilies analyzed in this study, CACTA has the fewest number of nTEs and the greatest number of cTEs (Figure 1, Additional file 1). This paucity of $n C A C T A s$ suggests that this superfamily generates fewer short elements than the others. Further, in three of the four genomes analyzed, CACTAs are enriched in intergenic regions where their copy numbers increase proportionally with genome size (see Figure 1). Finally, in at least two grass species (maize and rice) the presence of $n C A C T A s$ in or near genes has a negative correlation with transcription (Figure 4). Taken together, these data suggest that CACTA elements have either evolved a genic region insensitive/avoidance strategy or are removed from genic regions by selection.

The other four class 2 TE superfamilies also have distinctive features that are conserved in all genomes analyzed. For example, the ratio of the number of nTEs to cTEs is the highest for the Tc1/mariner superfamily and next highest for PIF/Harbinger followed by $h A T$, Mutator and CACTA (Figure 1). In this same order, the average length of nTEs also increases (Figure 2 and Additional file 2) suggesting that there is a range of lengths that is optimal for the transposition and amplification of each superfamily.

With regard to the distribution of elements in the four superfamilies, we have extended an observation originally made in rice [27] and show that except for $n C A C T A s$ nTEs are enriched at gene borders (Figure 3). Specifically, nTEs are most abundant at the $5^{\prime}$ gene border, and also enriched but less so near the $3^{\prime}$ border (Figure 3 and Additional file 3). This result, however, differs from a recent report in rice [18]. For the PIF/Harbinger superfamily, enrichment of the active MITE mPing was shown previously to result from its preference for insertion into gene proximal regions [26]. Although our data are descriptive and as such cannot distinguish between an insertion preference or winnowing by selection, the strikingly similar patterns around grass genes suggests a preference. A similar insertion preference was observed for Hermes, an active member of the $h A T$ superfamily from the housefly Musca domestica [28]. Characterization of almost two hundred thousand insertion sites in a Saccharomyces cerevisiae transposition assay revealed a marked preference for nucleosome free regions (NFRs) around genes, presumably because of their accessibility. Given that NFRs have also been found near the $5^{\prime}$ ends of plant genes [29] it is possible that their distribution underlies the pattern of nTE insertion sites from four of the five superfamilies in plants.

The dramatic enrichment of class 2 nTEs around genes especially in promoters prompted us to analyze the correlation between these elements and nearby gene expression levels using microarray data. Genes with PIF/ Harbinger and Tc1/mariner elements, which are the two superfamilies that generate the majority of MITEs, showed significantly higher expression values (Figure 4, Additional files 5 and 6). Furthermore, genes with $h A T$ and Mutators showed higher expression levels in maize but not in all rice tissues. In contrast, as discussed above, genes with CACTA elements were associated with lower gene expression.

\section{Conclusions}

These results indicate that superfamily identity needs to be considered when analyzing the correlation between TEs and the expression of nearby genes. This conclusion may explain discrepancies between our results and those of prior studies. For example, Hollister et al. and $\mathrm{Lu}$ et al. reported negative association between TEs and nearby genes in Arabidopsis [30] and rice [18], respectively. Based on our results, a possible explanation is that in both of these studies TEs were not separated into superfamilies for the analysis. Grouping of TEs in this way, without regard for superfamily identity, could mask the unique behavior of individual superfamilies. Finally, as our data and prior studies have shown, nTEs, especially MITEs, are abundant near genes [27]. It would be difficult to 
explain this distribution if the impact of these elements were largely negative.

\section{Methods}

Dataset

Genomic sequences and gene annotation results (version 1.0) of Brachypodium distachyon were downloaded from http://www.brachypodium.org [20]. Rice genome (build 5) was downloaded from http://rgp.dna.affrc.go.jp/, and gene annotation file (RAP3.gff3) was from http://rapdb.dna. affrc.go.jp [21,31]. Sorghum genome (Sorbi1) and annotations (Sbi1_4.gff) were downloaded from http://genome. jgi-psf.org/Sorbi1/Sorbi1.home.html [22]. Maize genome and annotations (version 4a53) were from http://www. maizesequence.org [1]. Microarray data files were downloaded from the Gene Expression Omnibus (GEO) database [32]. Rice RNA-seq data were downloaded from EBI (ERR008651, ERR008652, ERR008657, ERR008658, ERR008663 and ERR008664).

\section{Discovery and classification of class 2 TEs}

Class 2 TEs were discovered from genomic sequences as follows. Conserved transposase gene regions of putative autonomous TEs were identified using TARGeT [22]. Query sequences for TARGeT were curated from the conserved regions of known plant transposase sequences that were downloaded from Repbase [33]. Each transposase gene discovered in this way was considered to represent a single coding element (as listed in Additional file 1).

Noncoding elements were discovered using MITEHunter, which is a tool designed to specifically search for small noncoding class 2 TEs from genomic sequences [23]. MITE-Hunter outputs were manually checked using the approach described previously [23]. Qualified TE consensus sequences were classified into superfamilies based on their TIR sequences and TSD length using the following rules. Elements with TIRs starting with CACTA/G and with 3bp TSDs were identified as CACTA TEs, with 8bp TSDs as $h A T$ TEs, with 9 or 10bp TSDs as Mutator TEs, with $2 \mathrm{bp}$ TSDs that are TA as Tc1/mariner TEs and with 3bp TSDs that are TAA or TTA as PIF/Harbinger TEs. Nested TEs were removed and low complexity sequences were masked before homology searches by RepeatMasker described below.

Because one TE copy may mutate into several fragments, the number of total fragments is higher than the total copy number of TEs. We counted the nTE copy number using an approach similar to one introduced previously (see Additional file 1 , the $6^{\text {th }}$ column) [1]. Total length and copy numbers of class 2 noncoding TEs were determined using RepeatMasker as follows. Curated MITE-Hunter outputs were first masked by mdust (http://compbio.dfci.harvard.edu/tgi/software/) to filter low complexity regions and were used as library files for RepeatMaker (version 3.26, http://www.repeatmasker.org) to mask the genomic sequences with "nolow" and "-no_is" parameters. Because the positions of some TE copies overlap in the RepeatMasker output, to avoid counting a TE region twice, the original RepeatMasker output was first filtered and then the length and copy number of TEs were counted, which was done by a Perl script introduced in detail as follows. In the RepeatMasker output, if two consecutive TE copies overlapped, then the starting position of the second one was adjusted so it was right after the stop position of the first one. If a short TE copy was within a longer one, the shorter one was filtered. If a TE copy identified by RepeatMasker was missing less than 20bp from both ends of the query nTE consensus sequence, it was considered to be a double-ended copy. If a TE copy had only one end that was missing less than 20bp, it was counted as half of a copy. Other TE copies in the RepeatMasker output were considered as fragments that were not counted in the copy numbers. A nTE was considered to be a MITE if it was less than 600bp and had at least 25 double-ended copies, or 10 double-ended copies with an identity > $=99 \%$.

\section{Calculation of TE distribution within and around genes}

Several Perl scripts were written to acquire the genomic positions of TE regions and genes and to render figures of the distribution and proportion of TE sequences in and around genes. Positions of TE sequences were retrieved from processed RepeatMasker outputs. Positions of genes were retrieved from gene annotation files as follows. First, annotation files were checked to filter genes whose exons have contradicted directions. Genes inside other genes were also filtered. Second, for each gene, positions of different compartments were retrieved including $5^{\prime}$ flanking regions, $5^{\prime}$ UTR exons, $5^{\prime}$ UTR introns, exons, introns, $3^{\prime}$ UTR exons, $3^{\prime}$ UTR introns and $3^{\prime}$ flanking regions. Due to alternative splicing, one gene may have several sets of annotated compartments. In such cases, a combined gene model was generated using the following rules. If there was conflicted annotation information between two models, highest priority went to exons in the coding regions, with exons in the UTR next and introns with lowest priority. For example, a region annotated as an exon in one gene model but an intron in another was considered as an exon only.

For each type of gene compartment, an average percentage was calculated by dividing the total length of TE sequences within the gene compartment by the total length of the gene compartment. In the $5^{\prime} \mathrm{F}$ and the $3^{\prime} \mathrm{F}$ of all genes, TE sequence proportions were also calculated along each nucleotide position.

\section{Analyses of microarray expression data}

Gene expression values were acquired from microarray data in the GEO database. Rice data was from four sample 
series from two platforms: GPL6864 (Rice 44K, product of Agilent) and GPL2025 (Affymetrix Rice Genome Array). GPL6864 was designed based on RAP annotation results but GPL2025 was based on TIGR annotations. Because we used RAP annotations in this study, to use GPL2025 microarray data, we performed sequence comparisons between each GPL2025 probe sequence with RAP cDNA sequences. Maize expression data was from platform GPL10837 (Maize Pioneer Hi-Bred 105K mRNA Microarray). Because correlations between probe IDs and gene names in the gene annotation file were not known, as with the rice data, we performed comparisons between maize probes and maize cDNA sequences. For all microarray platforms, only genes whose probes had $100 \%$ identity to cDNA sequences were used. If a probe matched more than one gene its expression data was not used. If a gene had more than one probe, an average value was calculated.

\section{Analyses of RNA-seq data}

Reads were mapped to the rice reference genome using BWA [34]. PCR redundant reads were identified and filtered using picard (http://picard.sourceforge. net). Reads mapped within $80 \mathrm{bp}$ to the microarray probes were counted to represent the expression level of each gene.

\section{Statistical tests of the association between TEs and genes} Brunner-Munzel Test (Generalized Wilcoxon Test) was carried out using $\mathrm{R}$ package (version 2.12.0) to compare the expression levels between each TE-containing gene group with the control gene group. Genes (including $1 \mathrm{~kb}$ flanking regions) that contain the same superfamily of class 2 nTEs were put into the same group. Genes that did not have any class 2 nTEs were put into the control group. Only genes with at least $1 \mathrm{~kb}$ flanking region on either side were used in this analysis.

\section{Additional files}

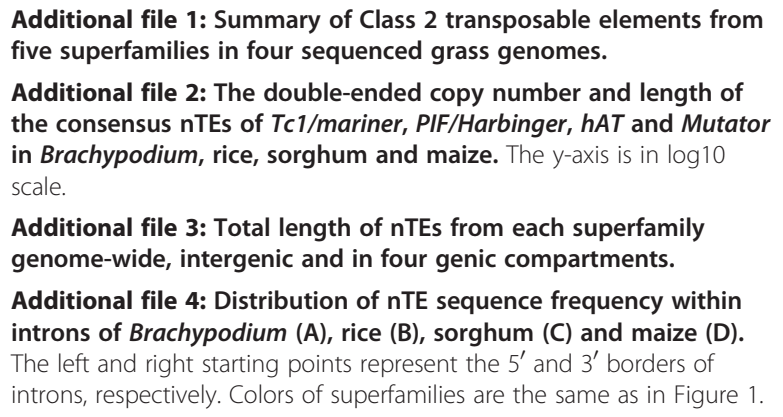

Additional file 5: Comparison of the expression of genes associated with different class 2 TE superfamily members vs. genes without class 2 TEs (control) (using microarray data).
Additional file 6: Comparison of the expression of genes associated with different class 2 TE superfamily members vs. genes without class 2 TEs (control) (using RNA-seq data).

\section{Competing interests}

The authors declare that they have no competing interests.

\section{Authors' contributions}

SRW and $\mathrm{YH}$ designed the experiments and wrote the manuscript. $\mathrm{YH}$ developed programs and performed the primary data analyses. SQ participated the analyses and performed the statistical tests. All authors read and approved the final manuscript.

\section{Acknowledgements}

We thank Yaowu Yuan, Aaron O. Richardson, Michael McKain, Xiaoyu Zhang and Jim Leebens-Mack for valuable discussions and suggestions. This study was supported by a grant from the NSF Plant Genome Program to SRW.

Received: 23 October 2012 Accepted: 29 January 2013

Published: 31 January 2013

\section{References}

1. Schnable PS, Ware D, Fulton RS, Stein JC, Wei F, Pasternak S, Liang C, Zhang J, Fulton L, Graves TA, et al: The B73 maize genome: complexity, diversity, and dynamics. Science 2009, 326(5956):1112-1115.

2. Nene V, Wortman JR, Lawson D, Haas B, Kodira C, Tu ZJ, Loftus B, Xi Z, Megy K, Grabherr M, et al: Genome sequence of Aedes aegypti, a major arbovirus vector. Science 2007, 316(5832):1718-1723.

3. Bennetzen JL: Transposable element contributions to plant gene and genome evolution. Plant Mol Biol 2000, 42(1):251-269.

4. Yuan YW, Wessler SR: The catalytic domain of all eukaryotic cut-and-paste transposase superfamilies. Proc Natl Acad Sci USA 2011, 108(19):7884-7889.

5. Feschotte C, Pritham EJ: DNA transposons and the evolution of eukaryotic genomes. Annu Rev Genet 2007, 41:331-368.

6. Wicker T, Sabot F, Hua-Van A, Bennetzen JL, Capy P, Chalhoub B, Flavell A, Leroy $\mathrm{P}$, Morgante M, Panaud $\mathrm{O}$, et al: A unified classification system for eukaryotic transposable elements. Nat Rev Genet 2007, 8(12):973-982.

7. Bureau TE, Wessler SR: Stowaway: a new family of inverted repeat elements associated with the genes of both monocotyledonous and dicotyledonous plants. Plant Cell 1994, 6(6):907-916.

8. Bureau TE, Wessler SR: Tourist: a large family of small inverted repeat elements frequently associated with maize genes. Plant Cell 1992, 4(10):1283-1294.

9. Wessler SR, Bureau TE, White SE: LTR-retrotransposons and MITEs: important players in the evolution of plant genomes. Curr Opin Genet Dev 1995, 5(6):814-821

10. Yang G, Nagel DH, Feschotte C, Hancock CN, Wessler SR: Tuned for transposition: molecular determinants underlying the hyperactivity of a Stowaway MITE. Science 2009, 325(5946):1391-1394.

11. Zhang X, Jiang N, Feschotte C, Wessler SR: PIF- and Pong-like transposable elements: distribution, evolution and relationship with Tourist-like miniature inverted-repeat transposable elements. Genetics 2004, 166(2):971-986.

12. Jiang N, Bao Z, Zhang X, Hirochika H, Eddy SR, McCouch SR, Wessler SR: An active DNA transposon family in rice. Nature 2003, 421(6919):163-167.

13. Feschotte C, Swamy L, Wessler SR: Genome-wide analysis of mariner-like transposable elements in rice reveals complex relationships with stowaway miniature inverted repeat transposable elements (MITEs). Genetics 2003, 163(2):747-758.

14. Kuang H, Padmanabhan C, Li F, Kamei A, Bhaskar PB, Ouyang S, Jiang J, Buell CR, Baker B: Identification of miniature inverted-repeat transposable elements (MITEs) and biogenesis of their siRNAs in the Solanaceae: new functional implications for MITEs. Genome Res 2009, 19(1):42-56.

15. Moreno-Vazquez S, Ning J, Meyers BC: hATpin, a family of MITE-like hAT mobile elements conserved in diverse plant species that forms highly stable secondary structures. Plant Mol Biol 2005, 58(6):869-886.

16. Yang G, Hall TC: MDM-1 and MDM-2: two mutator-derived MITE families in rice. $J$ Mol Evol 2003, 56(3):255-264.

17. Holyoake AJ, Kidwell MG: Vege and Mar: two novel hAT MITE families from Drosophila willistoni. Mol Biol Evol 2003, 20(2):163-167. 
18. Lu C, Chen J, Zhang Y, Hu Q, Su W, Kuang H: Miniature inverted-repeat transposable elements (MITEs) have been accumulated through amplification bursts and play important roles in gene expression and species diversity in Oryza sativa. Mol Biol Evol 2012, 29(3):1005-1017.

19. Fontanillas P, Hartl DL, Reuter M: Genome organization and gene expression shape the transposable element distribution in the Drosophila melanogaster euchromatin. PLOS Genet 2007, 3(11):e210.

20. Vogel JP, Garvin DF, Mockler TC, Schmutz J, Rokhsar D, Bevan MW: Genome sequencing and analysis of the model grass Brachypodium distachyon. Nature 2010, 463(7282):763-768.

21. Project IRGS: The map-based sequence of the rice genome. Nature 2005, 436(7052):793-800.

22. Paterson AH, Bowers JE, Bruggmann R, Dubchak I, Grimwood J, Gundlach H, Haberer G, Hellsten U, Mitros T, Poliakov A, et al: The Sorghum bicolor genome and the diversification of grasses. Nature 2009, 457(7229):551-556.

23. Han Y, Wessler SR: MITE-Hunter: a program for discovering miniature inverted-repeat transposable elements from genomic sequences. Nucleic Acids Res 2010,

24. Liao GC, Rehm EJ, Rubin GM: Insertion site preferences of the $P$ transposable element in Drosophila melanogaster. Proc Natl Acad Sci USA 2000, 97(7):3347-3351.

25. Zhang Q, Arbuckle J, Wessler SR: Recent, extensive, and preferential insertion of members of the miniature inverted-repeat transposable element family Heartbreaker into genic regions of maize. Proc Natl Acad Sci USA 2000, 97(3):1160-1165.

26. Naito K, Zhang F, Tsukiyama T, Saito H, Hancock CN, Richardson AO, Okumoto Y, Tanisaka T, Wessler SR: Unexpected consequences of a sudden and massive transposon amplification on rice gene expression. Nature 2009, 461(7267):1130-1134.

27. Zemach A, Kim MY, Silva P, Rodrigues JA, Dotson B, Brooks MD, Zilberman D: Local DNA hypomethylation activates genes in rice endosperm. Proc Natl Acad Sci USA 2010, 107(43):18729-18734.

28. Gangadharan S, Mularoni L, Fain-Thornton J, Wheelan SJ, Craig NL: Inaugural Article: DNA transposon Hermes inserts into DNA in nucleosome-free regions in vivo. Proc Natl Acad Sci USA 2010, 107 (51):21966-21972.

29. Kodama Y, Nagaya S, Shinmyo A, Kato K: Mapping and characterization of DNase I hypersensitive sites in Arabidopsis chromatin. Plant Cell Physiol 2007, 48(3):459-470.

30. Hollister JD, Smith LM, Guo YL, Ott F, Weigel D, Gaut BS: Transposable elements and small RNAs contribute to gene expression divergence between Arabidopsis thaliana and Arabidopsis lyrata. Proc Natl Acad SCi USA 2011, 108(6):2322-2327.

31. Tanaka T, Antonio BA, Kikuchi S, Matsumoto T, Nagamura Y, Numa H, Sakai $\mathrm{H}$, Wu J, Itoh T, Sasaki T, et al: The Rice Annotation Project Database (RAPDB): 2008 update. Nucleic Acids Res 2008, 36(Database issue):D1028-1033.

32. Barrett $\mathrm{T}$, Edgar R: Gene expression omnibus: microarray data storage, submission, retrieval, and analysis. Methods Enzymol 2006, 411:352-369.

33. Jurka J, Kapitonov W, Pavlicek A, Klonowski P, Kohany O, Walichiewicz J: Repbase Update, a database of eukaryotic repetitive elements. Cytogenet Genome Res 2005, 110(1-4):462-467.

34. Li H, Durbin R: Fast and accurate long-read alignment with BurrowsWheeler transform. Bioinformatics 2010, 26(5):589-595.

\section{Submit your next manuscript to BioMed Central and take full advantage of:}

- Convenient online submission

- Thorough peer review

- No space constraints or color figure charges

- Immediate publication on acceptance

- Inclusion in PubMed, CAS, Scopus and Google Scholar

- Research which is freely available for redistribution

Submit your manuscript at www.biomedcentral.com/submit 